\title{
RECUPERAÇÃO DOS COMPONENTES DO AROMA DA BEBIDA DE CAFÉ POR PERVAPORAÇÃO
}

\author{
ANDRÉ VON RANDOW ASSIS* \\ SÉRGIO HENRIQUES SARAIVA** \\ VIRGIINIAMARTINS DAMATTA***

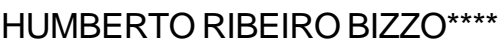 \\ LOURDES MARIA CORREAA CABRAL ${ }^{* * * * *}$
}

\begin{abstract}
Este trabalho teve como objetivo avaliar a pervaporação para a recuperação e concentração de compostos do aroma da bebida de café torrado e moído. Utilizou-se café torrado e moído de marca comercial como matéria-prima e membrana de etileno-propileno-dieno na pervaporação. O perfil aromático da bebida de café e do concentrado aromático foi caracterizado por cromatografia a gás e espectrometria de massa. Obteve-se extrato concentrado dos componentes do aroma de café, indicando que a pervaporação é capaz de concentrar o aroma da bebida de café. A membrana constituída pelo terpolímero etileno-propileno-dieno apresentou bom desempenho nesse processo.
\end{abstract}

* M.Sc. em Tecnologia de Alimentos, Universidade Federal Rural do Rio de Janeiro, Seropédica, RJ (e-mail: vonrandow@click21.com.br).

** D.Sc. em Tecnologia de Alimentos, Universidade Federal do Espírito Santo, Departamento Engenharia Rural, Alegre - ES (e-mail: sergio@cca.ufes.br).

*** D.Sc. em Tecnologia de Alimentos, Embrapa Agroindústria de Alimentos, Rio de Janeiro, RJ (e-mail: vmatta@ctaa.embrapa.br).

*** D.Sc. em Química Orgânica, Embrapa Agroindústria de Alimentos, Rio de Janeiro, RJ (e-mail: bizzo@ctaa.embrapa.br).

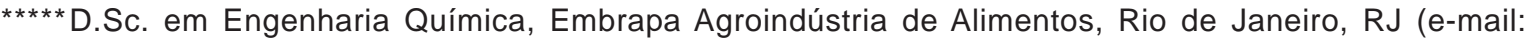
Icabral@ctaa.embrapa.br). 


\section{INTRODUÇÃO}

O Brasil é o maior produtor mundial de café, com participação superior a 33\% do mercado e produção anual superior a 30 milhões de sacas. É também o maior exportador e o segundo maior mercado consumidor, perdendo apenas para os Estados Unidos, havendo estimativa de tornar-se o primeiro mercado consumidor no ano de 2010 (ABIC, 2007).

A bebida de café apresenta aroma bastante agradável que atrai os consumidores. O processo de fabricação do café torrado é determinante na qualidade e, consequentemente, no aroma do produto final. O uso da temperatura no processo de torra resulta em transformações físicas e químicas nos grãos, promovendo a liberação de dióxido de carbono e outros produtos voláteis resultantes do processo de pirólise. Esses compostos serão responsáveis pelo aroma e sabor do café torrado. O teor dos componentes voláteis depende da matéria-prima utilizada, do processamento a que foi submetido o café verde, do grau de torra, da moagem e do procedimento usado no preparo da bebida (SEMMELROCH e GROSCH, 1996).

A fração volátil do café é muito complexa, sendo reportados na literatura mais de 800 compostos. Esses compostos são majoritariamente heterociclos, principalmente furanos (38-45\%), pirazinas (25-30\%), piridinas (3-7\%), e pirróis (2-3\%). Existem também fenóis, tiazóis e tiofenos (MOREIRA, TRUGO e DE MARIA, 2000). A partir da década de 60, com o advento da cromatografia a gás acoplada à espectrometria de massas (CG-EM), houve grande avanço na identificação de compostos voláteis do café, sendo que cerca de mil componentes já foram detectados (DE MARIA et al., 1996ab; BICCHI et al., 1997; AMSTALDEN, LEITE e MENEZES, 2001; MAEZTU et al., 2001; NASCIMENTO et al., 2003).

SARRAZIN et al. (2000) compararam cinco métodos diferentes de extração de aroma de café (extração supercrítica com dióxido de carbono, destilação e extração simultâneas, extração sobre pressão, arraste a vapor d'água e com solvente orgânico). A análise sensorial dos extratos indicou que 0 arraste a vapor foi o que apresentou o melhor resultado.

A pervaporação constitui processo de separação com membranas em que os componentes de uma mistura líquida permeiam através da membrana, sendo o permeado obtido na fase vapor (NEEL, 1991; HABERT, NOBREGA e BORGES, 2006). Tem sido citada como técnica com grande potencial de aplicação para a recuperação de compostos orgânicos voláteis a partir de meios líquidos, apontando dessa forma para a possibilidade da sua utilização na concentração de componentes de aroma (HUANG, MOON e PAL, 2002; KARLSSON e TRÄGÅRDH, 1996). Quando comparada com processos tradicionais de recuperação de aroma (tais como o arraste a vapor, a extração por solventes ou a extração supercrítica), a pervaporação apresenta como vantagens a ausência de solventes, a operação em condições amenas de temperatura e pressão, bem como o uso de sistemas compactos e modulares. Essas características conferem ao processo menor custo de operação e melhor qualidade do produto final, tendo em vista a não degradação térmica e a não contaminação do alimento por solventes orgânicos (RAJAGOPALAN e CHERYAN, 1995; OLSSON e TRÄGÅRDH, 1999; VAN HOOF et al., 2004; PEREIRA et al., 2005; ISCI, SAHIN e SUMNU, 2006; ASSIS et al., 2007).

PEREIRA et al. (2005) investigaram a pervaporação visando recuperar os componentes de aroma de sucos de frutas. Foram testadas membranas de polidimetilssiloxano (PDMS), do copolímero (etileno vinil acetato) (EVA) e do terpolímero etileno-propileno-dieno (EPDM). As membranas de EPDM apresentaram o melhor desempenho devido a sua baixa permeabilidade à água e alta seletividade aos componentes de aroma. A membrana de EPDM tem sido testada em várias aplicações da pervaporação quando o objetivo é concentrar compostos orgânicos hidrofóbicos, tais como componentes de aroma ou solventes organo-clorados (MEULEMAN et al., 2001).

O objetivo deste trabalho foi utilizar a pervaporação para extrair e concentrar compostos aromáticos característicos da bebida de café. 


\section{MATERIAL E MÉTODOS}

\subsection{MATÉRIA-PRIMAE MEMBRANAS}

A bebida de café foi preparada a partir do pó de café torrado e moído de marca comercial e utilizada como alimentação do processo de pervaporação. Para cada experimento, preparam-se $2 \mathrm{~L}$ da bebida de café de acordo com a informação descrita no rótulo da embalagem pelo fabricante (160 g de café torrado e moído para cada $2 \mathrm{~L}$ de água). A água foi aquecida até $95^{\circ} \mathrm{C}$, sendo, então, vertida sobre pó de café previamente colocado sobre filtro de papel.

Foram utilizadas membranas planas e densas, sintetizadas no Laboratório de Membranas da Coordenação dos Programas de Pós-Graduação em Engenharia da Universidade Federal do Rio de Janeiro (COPPE/UFRJ), a partir do terpolímero eteno-propileno-dieno (EPDM).

\subsection{PROCEDIMENTO EXPERIMENTAL}

Os experimentos foram realizados em sistema de pervaporação de bancada, constituído por dois módulos de permeação ligados em série, nos quais foram alocadas as membranas com área total de permeação de $0,0092 \mathrm{~m}^{2}$ (Figura 1).

A bebida de café foi continuamente bombeada sobre a superfície da membrana com auxílio de bomba centrífuga. Manteve-se a temperatura do processo constante, mediante banho termostático, sendo avaliadas as temperaturas de processo na faixa de $20^{\circ} \mathrm{C}$ a $45^{\circ} \mathrm{C}$. A diferença de pressão parcial foi garantida pelo uso de bomba de vácuo no lado do permeado. Recolheu-se o permeado obtido em condensador imerso em banho de nitrogênio líquido a $-196^{\circ} \mathrm{C}$ (ASSIS et al., 2007).

\section{FIGURA 1 - ESQUEMA DO SISTEMA DE PERVAPORAÇÃO}

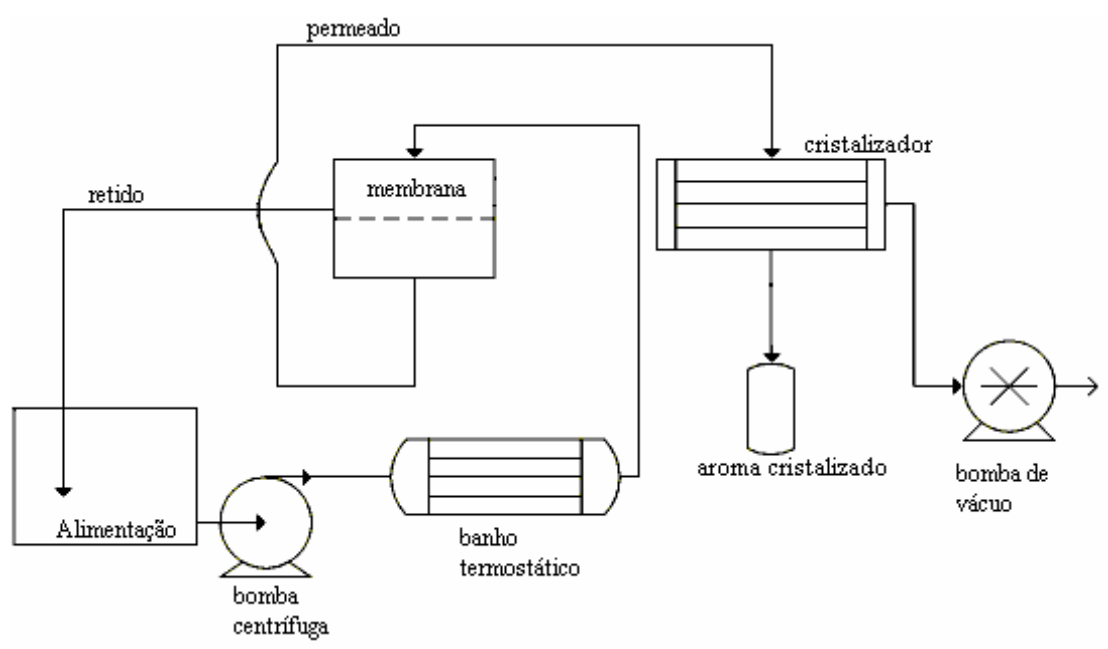

\subsection{MÉTODOS ANALÍTICOS}

Em todos os ensaios foram recolhidas amostras da bebida de café no tanque de alimentação antes (alimentação - A) e ao final do processo de pervaporação (retido - R) e do permeado (P) para posterior avaliação por cromatografia a gás acoplada à espectrometria de massas (CG-EM). Para a extração dos componentes voláteis das amostras antes da análise por CG utilizou-se a técnica de micro extração em fase sólida (solid phase micro extraction - SPME). Para tal, 0,3 $\mathrm{g}$ das amostras $(A, R, P)$ foram colocadas diretamente em frasco de $4 \mathrm{~mL}$ que permaneceram por 1 hora sob agitação em temperatura ambiente. Após esse tempo, fibra de SPME constituída de Divinilbenzeno/Carbaxen/PDMS (DVB/CAR/PDMS) foi exposta à fase vapor (headspace) da amostra por 15 minutos, sendo retraída e 
imediatamente levada ao cromatógrafo. A análise foi realizada em cromatógrafo Perkin Elmer $®$, modelo AutoSystem XL, utilizando-se detector de ionização de chama (FID) e coluna com fase estacionária de $5 \%$ fenil - 95\% metilpolissiloxano (HP-5), com 20 m de comprimento e 0,18 mm de diâmetro interno, com filme de $0,4 \mu \mathrm{m}$ de espessura. A programação de temperatura do forno foi de $40^{\circ} \mathrm{C}$ por 3 minutos, seguida de aquecimento até $240^{\circ} \mathrm{C}$ a $3^{\circ} \mathrm{C}$. min-1, permanecendo por $10 \mathrm{~min}$ a $240^{\circ} \mathrm{C}$. A fibra ficou exposta no injetor por três minutos, sendo o modo de injeção sem divisão de fluxo (splitless).

Os espectros de massas foram obtidos em sistema CG-EM, modelo Agilent 5973N, equipado com coluna com fase estacionária de 5\% fenil - 95\% metilpolissiloxano (HP-5MS) (30 m x 0,25 mm x $0,25 \mu \mathrm{m})$, utilizando-se hélio como gás de arraste $(1,0 \mathrm{~mL}$.min-1), nas mesmas condições descritas. Utilizou-se fonte de ionização do tipo Impacto de Elétrons (IE), com energia de ionização de $70 \mathrm{eV}$. Os índices de retenção foram calculados após a injeção de uma série de $n$-alcanos nas mesmas condições utilizadas para as amostras. A identificação dos constituintes foi realizada por comparação dos índices de retenção calculados com valores da literatura (ADAMS, 2001) e pela comparação dos espectros de massas obtidos com os da biblioteca Wiley (CG-MS).

\section{RESULTADOS E DISCUSSÃO}

Observou-se que o fluxo permeado apresentou correlação positiva com a temperatura (Figura 2). Essa observação está de acordo com o esperado, pois as etapas de sorção e a difusão na membrana sofrem influência da temperatura (NEEL, 1991).

Os cromatogramas das amostras da alimentação (bebida de café no início do processo), do retido (bebida de café ao final do processo) e do permeado (extrato concentrado) na pervaporação a $25^{\circ} \mathrm{C}$ estão ilustrados na Figura 3. Observa-se nitidamente a concentração de alguns componentes. $\mathrm{O}$ aumento no número de picos significa que a concentração de maior número de compostos ficou acima do limite de detecção do aparelho (Figura 3).

A escala do cromatograma da amostra da alimentação foi da ordem de grandeza de $10^{6}$, enquanto que no cromatograma da amostra de permeado a ordem de grandeza foi de $10^{8}$. Como a área é proporcional à concentração dos componentes pode-se dizer que a amostra de permeado está mais concentrada do que a amostra de alimentação. No cromatograma do permeado observou-se maior quantidade de picos em relação à amostra de alimentação e que os picos apresentam maior intensidade, indicando maior concentração daqueles compostos.

\section{FIGURA 2 - EFEITO DA TEMPERATURA SOBRE O FLUXO PERMEADO}

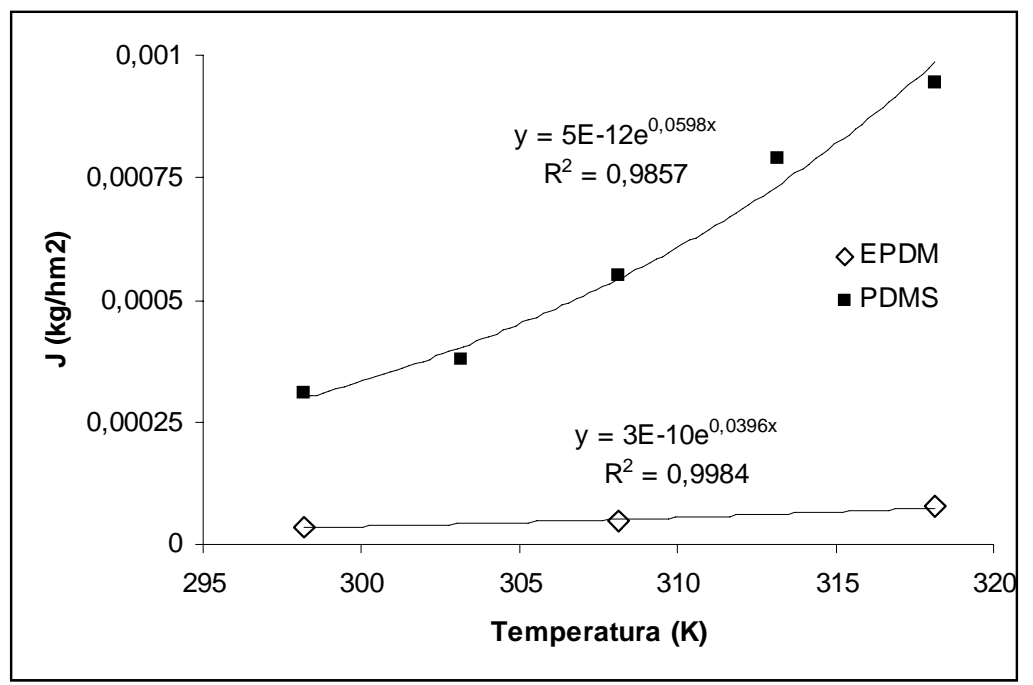


A ordem de grandeza das áreas dos picos nos cromatogramas da alimentação e do retido (alimentação ao final do processo) foi a mesma, o que pode indicar que não houve perda significativa dos componentes constituintes da bebida de café durante o processo.

Na Figura 4 são apresentados os cromatogramas de amostras da alimentação, do retido e do permeado de processo de pervaporação realizado a $35^{\circ} \mathrm{C}$ com a membrana de EPDM. Foram observados 36 picos no cromatograma da amostra da alimentação e 24 picos no cromatograma da amostra do retido. No cromatograma da amostra do permeado foram verificados 136 picos. Na análise de área total dos picos observou-se ordem de grandeza de $10^{6}$ para a alimentação, enquanto que no permeado a ordem de grandeza foi de $10^{8}$. A ordem de grandeza das áreas da alimentação e do retido apresentouse idêntica. Comparando a escala dos cromatogramas dos permeados obtidos a $25^{\circ} \mathrm{C}$ e $35^{\circ} \mathrm{C}$ não se constatou diferença, ambas apresentando valor na ordem de $10^{8}$.

\section{FIGURA 3 - CROMATOGRAMAS DA AMOSTRA DA ALIMENTAÇÃO (A), RETIDO (R) E PERMEADO (P) DO PROCESSO DE PERVAPORAÇÃO DA BEBIDA DE CAFÉ A $25^{\circ} \mathrm{C}$ COM A MEMBRANA DE EPDM E EXTRAÇÃO POR SPME}

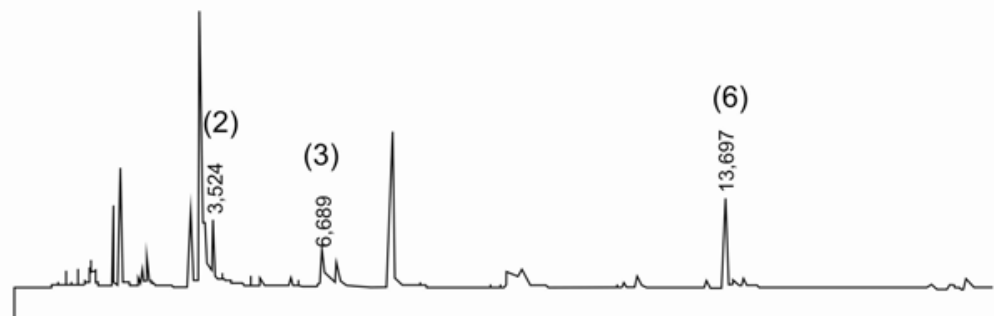

(A)

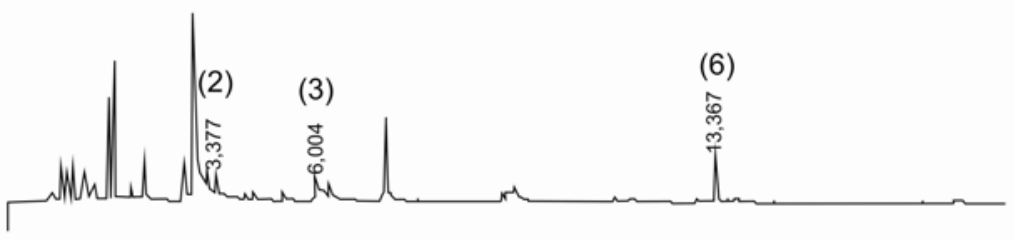

$(\mathrm{R})$

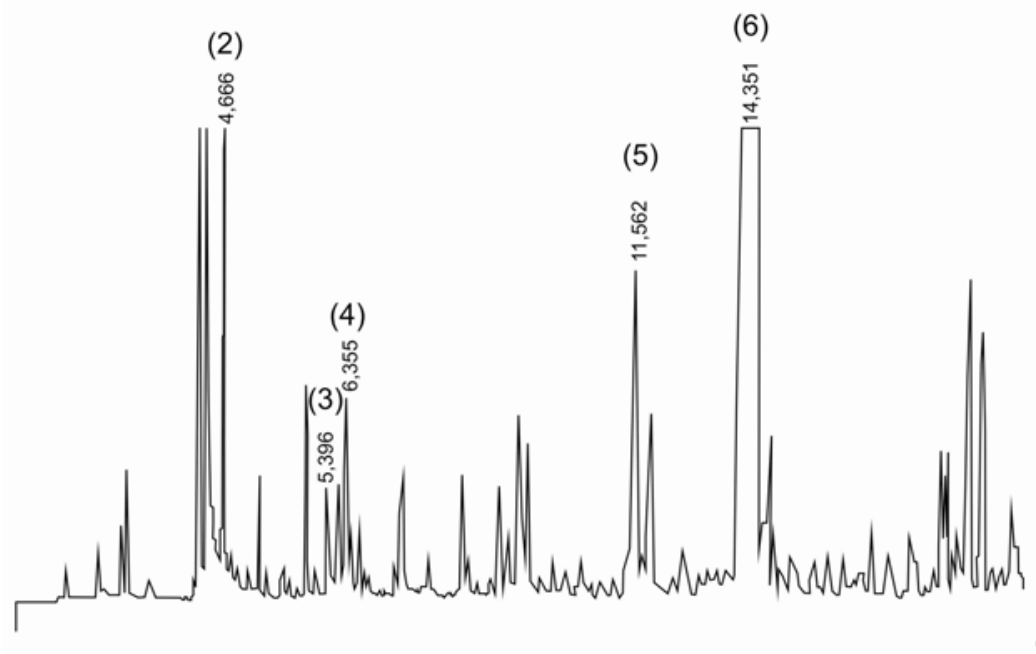

$(\mathrm{P})$

(1) 3-metilbutanal; (2)1-metil-1-pirrol; (3) Metilpirazina; (4) Furfural; (5)5-metil-2-furanocarboxialdeído; (6) 2-formil-1metilpirrol. 
Em todos os processos foi observada diminuição do número de picos do cromatograma da amostra do retido em relação ao da alimentação. Tal decréscimo foi mais elevado com maior temperatura do processo. O aumento da temperatura pode ter provocado maior perda de compostos voláteis da alimentação para o ambiente.

Entre os compostos detectados, 26 foram identificados nas amostras de alimentação e 52 nas amostras de permeado (Tabela 1). Dentre os compostos listados, 26 já foram citados na literatura consultada. ZAMBONIN et al. (2005) identificaram 32 componentes presentes em café em pó mediante SPME-CG-EM.

Observou-se, no presente trabalho, grande número de compostos heterocíclicos típicos de café, destacando-se os furanos, as pirazinas e os pirróis.

\section{FIGURA 4 - CROMATOGRAMAS DA AMOSTRA DA ALIMENTAÇÃO (A), RETIDO (R) E PERMEADO (P) DO PROCESSO DE PERVAPORAÇÃO DA BEBIDA DE CAFÉ A $35^{\circ} \mathrm{C}$ COM A MEMBRANA DE EPDM E EXTRAÇÃO POR SPME}

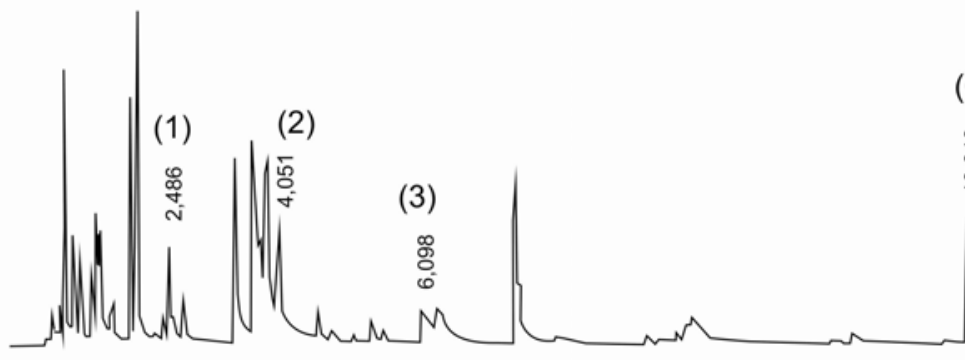

(6)

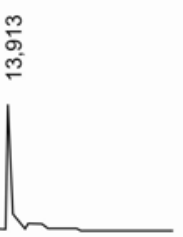

(A)

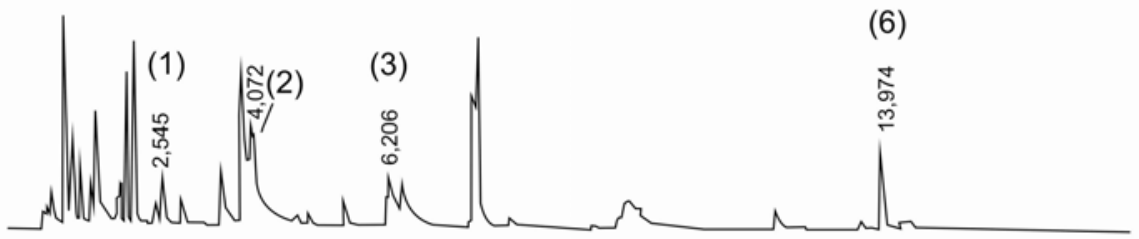

$(\mathrm{R})$

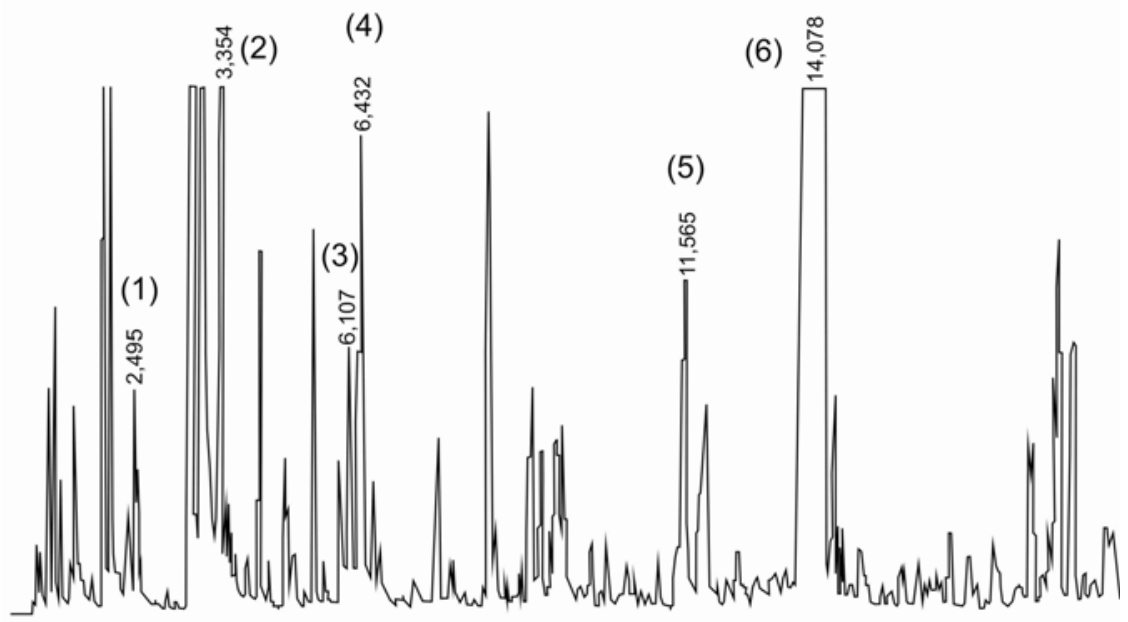

(1) 3-metilbutanal; (2)1-metil-1-pirrol; (3) Metilpirazina; (4) Furfural; (5)5-metil-2-furanocarboxialdeído; (6) 2-formil-1metilpirrol. 


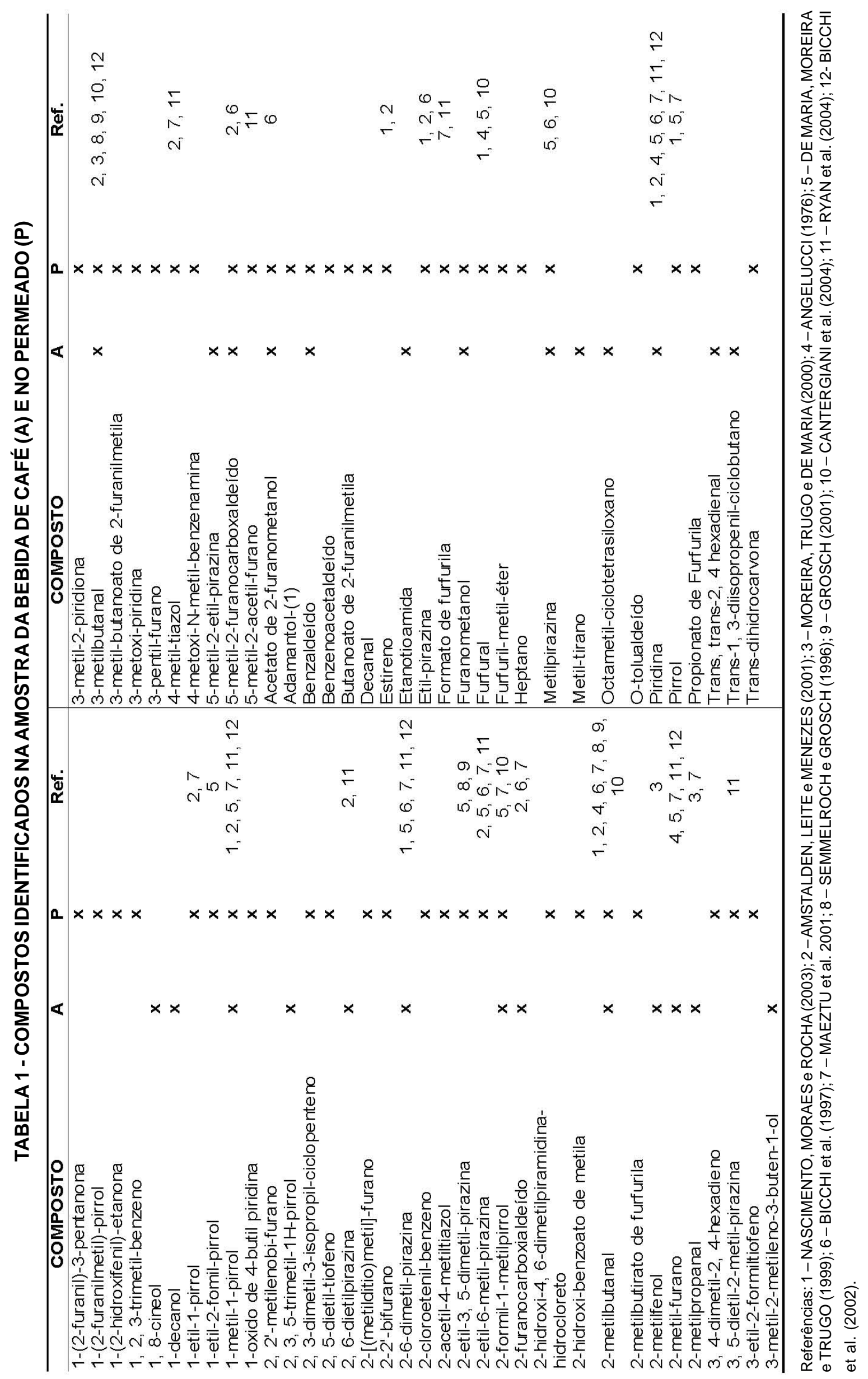


Os furanos são oriundos principalmente da degradação de glicídios presentes no café, contribuindo consideravelmente para as características sensorias do café torrado. A degradação pode ocorrer por meio de pirólise ou reação de Maillard (DE MARIA, MOREIRA e TRUGO, 1999). Dentre os furanos detectados pode-se citar o 2-metil furano, que fornece aroma similar ao de guaraná e que foi identificado somente na alimentação, e o furfural que apresenta aroma de grama, verificado apenas no permeado.

As pirazinas são compostos característicos de produtos submetidos a tratamento térmico. A 2-etil-3,5-dimetil pirazina representa composto forte no aroma de café, já a 2-etil-6-metil pirazina não é considerada como substância relevante para o aroma de café (DE MARIA, MOREIRA e TRUGO, 1999). Essas duas substâncias foram identificadas nas amostras de permeado analisadas. Das pirazinas identificadas pode-se destacar a 2-6-dimetil-pirazina, substância de grande utilidade no monitoramento do grau de torra do café, segundo estudo realizado por HASHIM e CHAVERON (2006).

As amostras da bebida de café (tanto de alimentação quanto permeado) apresentaram aspecto e aroma característico (Figura 5). A amostra de permeado apresentou aspecto transparente e aroma de café. A coloração transparente do permeado deve-se ao fato de que somente os componentes voláteis do café permeiam através da membrana de pervaporação, sendo retidos os componentes responsáveis pela coloração.

\section{FIGURA 5 - AMOSTRAS DO PROCESSO DE PERVAPORAÇÃO}

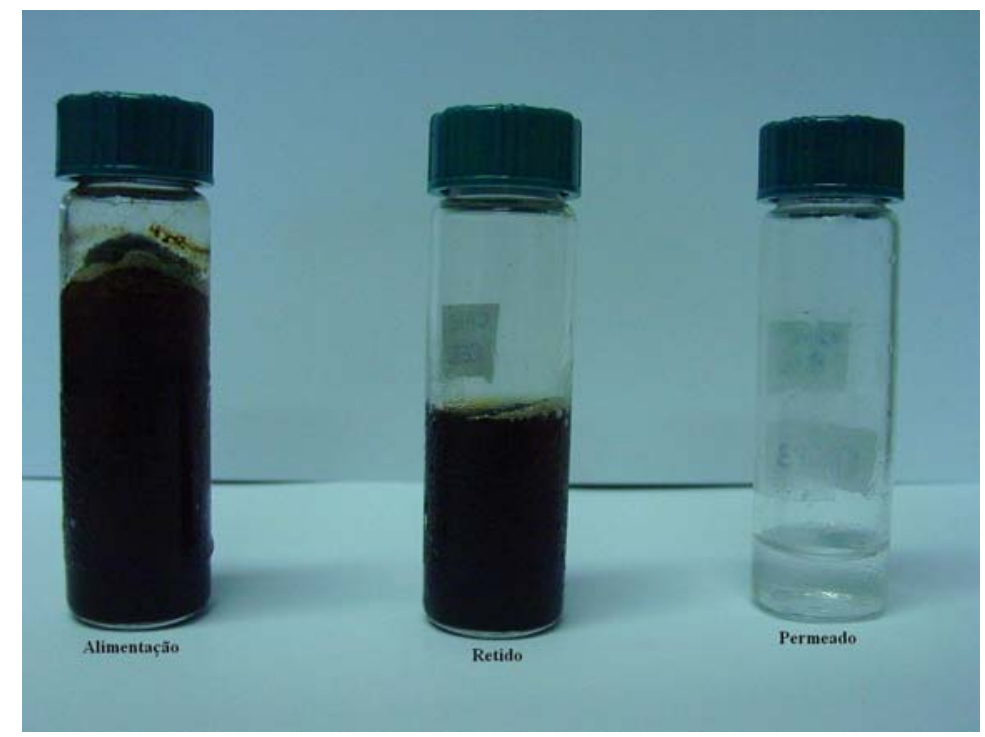

\section{CONCLUSÃO}

A pervaporação constitui técnica promissora para recuperar e concentrar os compostos do aroma de café.

As substâncias responsáveis pela cor, bem como a cafeína foram retidos pela membrana. Ao contrário, os principais componentes do aroma que contribuem para o aroma característico do café permearam através da membrana e foram concentrados.

A membrana de EPDM apresentou bom desempenho e pode ser apontada como opção para a pervaporação de bebida de café.

\section{ABSTRACT}

\section{RECOVERY OF THE AROMA COMPOUNDS FROM COFFEE BEVERAGE BY PERVAPORATION PROCESS}

The objective of this work was to evaluate the pervaporation for recuperation and concentration of aroma compounds of roasted and ground coffee beverage. Roasted and ground coffee of commercial brand was used as raw material and membranes of ethylene-propylene-diene terpolymer were used in pervaporation. The aromatic profile of the coffee beverage was determined by gas chromatography and mass spectrometry. 
It was obtained a concentrated extract rich in the aroma compounds of coffee beverage showing that pervaporation is capable to concentrate coffee beverage aroma. The membrane formed of ethylenepropylene-diene terpolymer exhibited a good performance during such a process.

KEY-WORDS: CONCENTRATED AROMATIC EXTRACT; MEMBRANE PROCESS; GAS CHROMATOGRAPHY; Coffea arabica; Coffea robusta.

\section{REFERÊNCIAS}

1 ABIC. Associação Brasileira da Indústria do Café. Indicadores de desempenho da cafeicultura brasileira. Disponível em: <http://www.abic.com.br/estat_desempenho.html>. Acesso em: 4 maio 2007.

2 ADAMS, R. Identification of essential oils by gas chromatography/mass spectrometry, $4^{\text {th }}$ ed. Carol Stream, Illinois: Allured Publishing, 2001. 804 p.

3 AMSTALDEN, L. C.; LEITE, F.; MENEZES, H. C. Identificação e quantificação de voláteis de café através de cromatografia gasosa de alta resolução/espectrometria de massas empregando um amostrador automático de "headspace". Ciência \& Tecnologia de Alimentos, São Paulo, v. 21, p. 123-128, 2001.

4 ANGELUCCI, E. Aroma em café e derivados. In: FIGUEIREDO, I. B.; UZELAC, M. (Ed.). Aromas em alimentos. Campinas-SP: ITAL, 1976. p. 123-139. (ITAL- Instruções Técnicas, 9)

5 ASSIS, A.R.; BIZZO, H.R.; MATTA, V.M.; CABRAL, L.M.C. Recuperação e concentração de componentes do aroma de caju (Anacardium occidentale L.) por pervaporação. Ciência \& Tecnologia de Alimentos, São Paulo, v.27, n.2, p.349-354, 2007.

6 BICCHI, C. P.; PANERO, O.M.; PELLEGRINO, G.M.; VANNI, A.C. Characterization of roasted coffee and coffee beverages by solid phase microextration-gas chromatography and principal component analysis. Journal Agricultural and Food Chemistry, v. 45, p. 4680-4686, 1997.

7 BICCHI, C.; IORI, C.; RUBIOLO, P.; SANDRA, P. Headspace sorptive extraction (HSSE), stir bar sorptive extraction (SBSE), and solid phase microextraction (SPME) applied to the analysis of roasted Arabica coffee and coffee brew. Journal Agricultural and Food Chemistry, v.50, n.3, p.449-59, 2002.

8 CANTERGIANI, E.; BREVARD, H.; KREBS, Y.; FERIA-MORALES, A.; AMADÒ, R.; YERETZIAN, C. Characterization of the aroma of green Mexican coffee and identification of mouldy/earthy defect. European Food Research and Technology, v. 212, n.6, p.648-657, 2004.

9 DE MARIA, C. A. B.; TRUGO, L.C.; MOREIRA, R.F.A.; WERNECK, C.C. Composition of green coffee water-soluble fractions and identification of volatiles formed during roasting. Food Chemistry, v. 55, p. 203-207, 1996 a.

10 DE MARIA, C. A. B; MOREIRA, R. F. A.; TRUGO, L. C. Componentes voláteis do café torrado. Parte I: compostos heterocíclicos. Química Nova, v. 22, p. 209-217, 1999.

11 DE MARIA, C.A.B.; TRUGO, L.C.; AQUINO NETO, F.R.; MOREIRA, R.F.A.; ALVIANO, C.S. The CG/MS identification of volatiles formed during the roasting of high molecular mass coffee aroma precursors. Journal of Brazilian Chemistry Society v. 7, p.267-270, 1996b.

$12 \mathrm{GROSCH}$, W. Evaluation of the key odorants of foods by dilute experiments, aroma models and omission. Chemical Senses, v. 26, p.533-545, 2001.

13 HABERT, A C; NOBREGA, R.; BORGES C. Processos de separação com membranas. Rio de Janeiro: E-papers, 2006. 180 p.

14 HASHIM, L.; CHAVERON, H. Evaluation of sensory attributes of coffee brews from robusta coffee roasted under different conditions. European Food Research and Technology, v.224, n.2, p.159-165, 2006.

15 HUANG, R. Y. M.; MOON G. Y.; PAL, R. Ethylene propylene diene monomer (EPDM) membranes for the pervaporation separation of aroma compouds from water. Ind. Engineering Chemistry Reserch, v.41, p. 531-537, 2002.

$16 \mathrm{ISCl}$, A.; SAHIN, S.; SUMNU, G. Recovery of strawberry aroma compounds by pervaporation. Journal of Food Engineering, v. 75, p. 36-42, 2006.

17 KARLSSON, H. O. E.; TRÄGÅRDH, G. Applications of pervaporation in food processing - Review. Trends in Food Science \& Tecnology, v. 7, p. 78-83, 1996.

18 MAEZTU, L.; SANZ, C.; ANDUEZA, S.; DE PENA, M.P.; BELLO, J. Characterization of espresso coffee aroma by static headspace gc-ms and sensory flavor profile. Journal of Agricultural Food Chemistry, v, 49, n.11, p.5437-5444, 2001. 
19 MEULEMAN, E. E. B.; WILLEMSEN, H.A.; MULDER, M. H. V.; STRATHMANN, H. EPDM as a selective membrane material in pervaporation. Journal of Membrane Science, v.188, p. 235-249, 2001.

20 MOREIRA, R.F.A.; TRUGO, L.C.; DE MARIA, C.A.B. Componentes voláteis do café torrado. Parte II. Compostos alifáticos, alicíclicos e aromáticos. Química Nova, v.23, n.2, p.195-203, 2000.

21 NASCIMENTO, E. A.; MORAIS, S. A. L.; ROCHA, R. S. Constituintes voláteis de cafés "gourmet" e mole do cerrado do triângulo mineiro em função da torra. Ciência e Tecnologia de Alimentos, São Paulo, v. 23, p. 282-284, 2003.

22 NEEL, J. Introduction to pervaporation. In: HUANG, R.Y.M. (Org.). Pervaporation membranes separation processes. Amsterdam: Elsevier Science Publishers, 1991. p. 1-109.

23 OLSSON, J.; TRÄGÅRDH, G. Influence of temperature on membrane permeability during pervaporation aroma recovery. Separation Science and Tecnology, v. 34, p. 1643-1648, 1999.

24 PEREIRA, C.C.; RUFINO, J.R.M.; HABERT, A.C.; NOBREGA, R.; CABRAL, L.M.C.; BORGES, C.P. Aroma compounds recovery of tropical fruit juice by pervaporation: membrane material selection and process evaluation. Journal of Food Engineering, v.66, p.77-87, 2005.

25 RAJAGOPALAN, N.; CHERYAN, M. Pervaporation of grape juice aroma. Journal of Membrane Science, v. 104, p. 243-250, 1995.

26 RYAN, D.; SHELLIE, R.; TRANCHIDA, P.; CASILLI, A.; MONDELLO, L.; MARRIOTT, P. Analysis of roasted coffee bean volatiles by using comprehensive two-dimensional gas chromatography-time-of-flight mass spectrometry. Journal of Chromatography A, v. 1054, p. 57-65, 2004.

27 SARRAZIN, C.; LE QUÉRÉ, J.L.; GRETSCH, C.; LIARDON, R. Representativeness of coffee aroma extratcs: a comparison of differets extraction methods. Food Chemistry, v. 70, p. 99-106, 2000.

28 SEMMELROCH, P.; GROSCH, W. Studies on character impact odorants of coffee brews. Journal of Agricultural and Food Chemistry, v. 44, p. 537-543, 1996.

29 VAN HOOF, V.; VAN DEN ABEELE, L.; BUEKENHOUDT, A; DOTREMONT, C.; Economic comparison between azeotropic distillation and different hybrid systems combining distillation with pervaporation for the dehydration of isopropanol. Separation and Purification Technology, v.37, n.1, p.33-49, 2004.

30 ZAMBONIN, C.G.; BALEST, L.; DE BENEDETTO, G.E.; PALMISANO, F. Solid-phase microextraction-gas chromatography mass spectrometry and multivariate analysis for the characterization of roasted coffees. Talanta, v. 66, p. 261-265, 2005.

\section{AGRADECIMENTOS}

Ao Consórcio Brasileiro de Pesquisa e Desenvolvimento do Café pelo suporte financeiro ao projeto. Ao CNPq pela bolsa de mestrado de André von Randow de Assis. A equipe agradece especialmente ao Prof. Cristiano P. Borges, do Laboratório de Membranas da COPPE/UFRJ pelo apoio na síntese das membranas de EPDM. 